\title{
Estimation of Serum Chemerin, Visfatin Levels and Their Ratio as a Possible Diagnostic Parameters of Rheumatoid Arthritis
}

Dunia M. Mohammed Ali ${ }^{1}$, Suhaer Zeki Al-Fadhel ${ }^{2}$, Nibras H. Abdulsada Al-

Ghuraibawi ${ }^{3}$, Hussein Kadhem Al-Hakeim ${ }^{4}$

1: Department of Biochemistry, College of Medicine, University of Kufa, Iraq. E-mail: duniam.mohammedali@uokufa.edu.iq

2: Department of Clinical Laboratory Science, College of Pharmacy, University of Kufa, Iraq. E-mail: suhairz.saeed@uokufa.edu.iq

3. Department of Pharmacology, College of pharmacy, University of Kufa, Iraq. E-mail: nibrash.abdalsada@uokufa.edu.iq

4: Department of Chemistry, College of Science, University of Kufa, Iraq. E-mail: headm2010@yahoo.com.

Corresponding author: Prof.Dr.Hussein Kadhem Al-Hakeim, Department of Chemistry, College of Science, University of Kufa, Iraq. E-mail: headm2010@yahoo.com. 


\begin{abstract}
Objective: Rheumatoid Arthritis (RA) is a chronic inflammatory disease affecting the synovium and articular cartilage that initiates joint damage. RA associates with a change in many inflammatory biomarkers. The present study aims to measure the levels of inflammatory adipocytokines (chemerin and visfatin) and their ratio in addition to some related biomarkers in RA patients group in comparison to healthy control group and find out their efficacy in diagnosis of RA.

Methods: The study included 60 RA patients and 30 healthy control group. Serum visfatin and chemerin were measured by using ELISA technique, while other biomarkers were determined spectrophotometrically. Multivariate general linear model analysis and Receiver operating curve was used to study the opportunity of using chemerin and visfatin as diagnostic tools for RA.

Results: The results indicated that there was a significant increase $(\mathrm{p}<0.05)$ in all lipid profile components, except HDLc that decreased, in RA patients in comparison with healthy control group; while significant decrease $(\mathrm{p}<0.001)$ in high density lipoproteincholesterol of RA patients in comparison with control group. Serum chemerin, visfatin, Creactive protein, and uric acid levels were significantly higher $(\mathrm{p}<0.05)$ in RA patients than those of control group. The results showed a relatively good sensitivity and specificity of chemerin (sensitivity $=88.1$, specificity $=75.9$ ) at a concentration $=187.88 \mathrm{ng}$ in diagnosis of RA.

Conclusions: chemerin is able to diagnose RA efficiently but this diagnoses, due to relatively small specificity, may interfere with other inflammatory disorders. However, with adjuvant with other diagnostic parameters, chemerin may represent a useful addition in diagnosis of RA.
\end{abstract}

Key Words: Rheumatoid Arthritis; chemerin; visfatin; CRP; uric acid. 


\section{Introduction}

Rheumatoid Arthritis (RA) is a systemic autoimmune disease characterized by synovitis and joint erosion in addition to extra articular manifestations include pulmonary, and vascular disease were appeared. It affects $\sim 0.5-1 \%$ of the population. ${ }^{1}$ In a female/male ratio ranging from $2: 1$ to $4: 1, \mathrm{RA}$ an insidious disease, typically having an onset of symmetric joint swelling and reaching a peak incidence in the fourth and fifth decades. ${ }^{2-3}$ The etiology of RA remains uncertain but some studies that belong to environmental and genetic factors were responsible. ${ }^{4} \mathrm{RA}$ was a long term autoimmune disorder that primarily affects joints. ${ }^{5}$ It was typically manifest with signs of inflammation, with the affect joints being swollen, warm, painful and stiff, particularly early in the morning on waking or following prolonged inactivity. ${ }^{6} \mathrm{RA}$ represents the most common form of autoimmune diseases and immune cell infiltrates are observed in synovial tissues of RA patients. ${ }^{7}$ The production of certain inflammatory markers by different cells such as monocytes/macrophages, fibroblast-like synoviocytes, dendritic cells plays a central role in promoting and maintaining joint inflammation. ${ }^{8}$ There is a continuous need for a better diagnostic biomarkers for early identification of RA. Studies were focused on some trace elements ${ }^{9}$ and various proteins. ${ }^{10}$ One of the possible future proteins is the inflammatory biomarkers is chemerin. It is a $16 \mathrm{kDa}$ protein purified from ascetic fluids of ovarian cancer patients and synovial exudates of RA patients. Chemerin revealed anti-inflammatory and pro-inflammatory effects by binding with the CMKLR1 receptor. ${ }^{11}$ It plays a role in the differentiation of adipocytes and glucose metabolism. ${ }^{12}$ Recently it was identified as one of the adipokines with autocrine, paracrine and even endocrine roles in vivo. ${ }^{13}$ Chemerin also is involved in innate and adaptive immunity as a chemoattractant for natural killer cells, macrophages, and certain dendritic cell subsets, ${ }^{14}$ in addition to also as an adipokine. ${ }^{15,16}$ The term adipokine was apply to all the substances have biological activity synthesized by White adipose tissue (WAT) which function as regulator of energy homeostasis and metabolism; the same mediators were also involved in chronic inflammation and metabolic dysfunctions. ${ }^{17}$ WAT was known as a pleiotropic organ specialized in endocrine functions being capable to create several hormones and other proteins involved in both physiological and pathological processes, as well as immunity and inflammation. ${ }^{18}$ Obesity is a risk factor for autoimmune diseases as RA ${ }^{9,} 19$ since adipose tissue releases adipokines able to create a low grade inflammatory environment. ${ }^{20}$ Recently, Chemerin has emerged as a key adipokine involved in immune response. 
Overweight and obese healthy subjects show higher Chemerin values than normal weight, whose plasma values decrease after diet. ${ }^{21,22}$

Another pro-inflammatory adipokine that potentiate to play a role in diagnosis of RA is visfatin; a pre-B cell colony-enhancing factor involved in early B-cell development and acts as a nicotinamide phosphoribosyl transferase secreted mainly by visceral fat. ${ }^{23}$ It is also seen in liver, skeletal muscles and bone marrow and produced by visceral WAT, which mimics the effects of insulin. ${ }^{24}$ The pro-inflammatory action of visfatin was describe to be mediated by insulin signaling pathway ${ }^{25}$ and one research showed an increase of serum visfatin levels in patients with RA. ${ }^{26}$ Nowadays, the significant role of adipocytokines such as chemerin and visfatin in mediating joint damage have been indicated by different studies. ${ }^{27}$ The goal of the present study is to measure the level of serum chemerin and visfatin, in addition to their relationship in RA patients and calculate their ability to be a future diagnostic tools for RA. The factors that may affect their levels (lipid profile, Uric acid (UA), body mass index (BMI), and C-reactive protein (CRP)) were also studied by using correlation studies and multivariate general linear model (GLM) analysis.

\section{Materials and Methods}

This case-control study was conducted in Al-Sader Teaching Hospital in Al-Najaf province-Iraq, during the period from January 2019 to March 2019. The present study included 60 RA patients and 30 healthy control group. The ages of RA patients and the control groups were matched and ranged from 20-60 years old. RA patients were diagnosed according to the criteria putted in 2010 by American College of Rheumatology and the European League against Rheumatism. ${ }^{28}$ All patients were achieved a total score more than 6 from the 4 domains: the number and site of involved joints, serologic abnormalities (presence of rheumatoid factor or anti-citrullinated peptide/protein antibody), elevation of inflammatory markers (erythrocyte sedimentation rate and/or CRP, and the duration of symptoms. Patients were evaluated by full medical history to exclude any existing systemic disease that may affect the parameters to be diagnosed, particularly diabetes, liver disease, and renal disease; otherwise the patient was excluded from the study. Subjects suffered from any disease which interferes with the obtained were excluded. Body mass index (BMI) was measured by dividing weight in kilograms by length of subjects in square meter. ${ }^{29}$ Approval for the study was obtained from the ethics committee of the University 
of Kufa, Iraq (111/2018), which is in compliance with the International Guideline for Human Research protection as required by the Declaration of Helsinki.

Seven milliliters of fasting venous blood samples were collected from patients and control group after overnight fasting and allowed to clot at $37^{\circ} \mathrm{C}$ then centrifuged at $3000 \mathrm{rpm}$ for $15 \mathrm{~min}$. Sera were removed and frozen at $-20^{\circ} \mathrm{C}$ until analysis. Serum chemerin and visfatin were determined by ELISA assay supplied by Elabscience, USA. Serum lipid profile (TC, TG, LDLc, VLDLc and HDLc), CRP and uric acid levels were determined by the colorimetric method using kits.

\section{Statistical analysis}

The distribution of the results were examined using Kolmogorov-Smirnov test and found as normal statistical distribution for all the measured parameters. All results were expressed as mean \pm standard deviation. Pooled $\mathrm{t}$-test has been used for the comparison between the patients and control groups and among subdivided groups in the measured parameters. Pearson's correlation coefficients (r) were calculated to estimate the correlation between parameters. The general linear model (GLM) analysis was used to assess the effects of diagnosis while controlling for confounding variables including BMI and age. Receiver Operating Curve (ROC) was used to study the opportunity of using Chemerin, visfatin, and their ratio as a diagnostic tools for RA. The difference between groups is considered as statistically different when $\mathrm{p}<0.05$. All statistical analysis were performed using SPSS Statistics Version 25 (2017) by IBM-USA.

\section{Results}

\section{Socio-demographic, clinical and biomarker characteristics}

The socio-demographic and clinical data of the RA patients and healthy control groups are presented in Table 1. The results show no significant difference in age or BMI in RA group in comparison with control group. CRP and UA were also increase in patients indicated the involvement of inflammation and high uric acid in the inflammatory response of the patients. Visfatin, Chemerin and their ratios also increased in patients as compared with controls. There is a significant increase in all lipid profile components except HDLc which decreased in patients as compared with controls. Atherogenic indices (TC/HDLc, TG/HDLc and LDLc/HDLc) were increase which revealed a susceptibility to atherosclerosis and heart diseases. 


\section{Multivariate GLM analysis with the biomarkers}

The results of multivariate GLM analysis with the biomarkers as dependent variables and diagnosis as explanatory variable were demonstrated in Table 2. The analysis showed a high effect of being RA patients on the all measured biomarkers i.e., $70.7 \%$ of the change in the level of measured biomarkers can be explained by the presence of RA disease (Partial $\left.\eta^{2}=0.707, \mathrm{p}<0.001\right)$. Age and BMI have a small and statistically insignificant effect on the measure parameters (Partial $\eta^{2}=0.075, \mathrm{p}=0.071$ ), and (Partial $\eta^{2}=0.107, \mathrm{p}=0.054$ ), respectively. To explore which biomarker affected by diagnosis, we perform the tests of between subjects affect in the second part of the table. The results showed that all biomarkers affected significantly be the diagnosis and the higher effects where on CRP (Partial $\eta^{2}=0.480, p<0.001$ ), Chemerin (Partial $\eta^{2}=0.295, \mathrm{p}<0.001$ ), and UA (Partial $\left.\eta^{2}=0.252, p=0.071\right)$. While visfatin showed Partial $\eta^{2}=0.079$ only.

\section{Correlation between the parameters}

The results in Table 3 indicate that visfatin was correlated positively with chemerin and a negatively with chemerin to Visfatin ratio. There was also a positive correlation between Chemerin and TC, HDLc, LDLc, LDLc/HDLc, CRP and UA, while it was negatively correlated with HDLc. These results indicated a relationship between chemerin and lipid profile and inflammation status. CRP show a positive correlation with UA, BMI, Chemerin/Visfatin, TG, VLDLc, TC/HDLc, TG/HDLc and LDLc/HDLc, while it was negatively correlated with HDLc. UA has a positive correlation with TG/HDLc and a negative correlation with HDLc. Regarding the age, the results indicate a negative correlation only with Chemerin/Visfatin.

\section{Receiver Operating Curve (ROC) as a Diagnostic tool for RA}

Results of the ROC curve and AUC analysis for the Visfatin and chemerin in addition of their ratio as a possible diagnostic parameters for RA are presented in Figure 1 and Table 4 . The results showed a relatively good sensitivity and specificity of chemerin ( sensitivity $=88.1$, specificity $=75.9$ ) at a concentration=187.88ng in diagnosis of RA in comparison with visfatin (sensitivity $=64.4$, specificity $=58.6$ ) at a concentration=39.02 and the chemerin/visfatin ratio ( sensitivity $=66.1$, specificity $=65.5$ ) at a cut-off value $=5.03$. The p-values of the AUC are less than 0.05 and statistically significant. The Youdin J statistics of the parameters in Figure 1 confirms these results. 


\section{Discussion}

The major finding in the present study is the significant increase in chemerin and visfatin levels in addition to a significant increase in UA, CRP, TC, TG, LDLc, VLDLc of RA patients when compared with healthy control as seen in Table 1. While HDLc was significantly decreased in the RA patients in comparison to the control group. These results indicated a state of dyslipidemia and inflammation in RA disease. Regarding dyslipidemia, adipose excess is an additional extra-articular feature of RA ${ }^{30}$ and the increase in circulating lipid concentrations may make RA patients at higher of developing a cardiovalscular diseases. ${ }^{31,32}$ These adipose tissues also secrete many adipokines including visfatin and chemerin. Very few studies have addressed adipokine in RA. ${ }^{33}$ Adipokines secreted predominantly by WAT are involved in the pathogenesis of different RA, having potent modulatory effects on synovial, cartilage, bone and immune cells. ${ }^{34,35}$ Several researches had exposed its role in metabolic syndrome, liver disease, chronic kidney disease, cancer, obesity and diabetes. ${ }^{36-39}$ Chemerin was involved in innate and adaptive immune response, firstly codified in it was precursor low biological active form. ${ }^{40,41}$ Once activated, it was trigger rapid defenses in the body by directing dendritic cells and macrophages to injured tissues and inflammation sites. Serum adipokine concentrations were connected with obesity, diabetes, lipid profile components, and premature vascular inflammation. ${ }^{42}$ Chemerin and its receptor CMKLR1 form a complex involved in the regulation of immune response which can contribute to both the onset and termination of acute inflammation. ${ }^{43}$ Elevation of chemerin level can directly favor inflammation by recruiting immune system cells. ${ }^{44}$ Chemerin also increased expression and secretion of inflammatory mediators to the inflamed spot. ${ }^{45}$ Increased concentrations of this adipokine in adipose tissue cause the recruitment of immune cells, consequently elevated the expression of inflammatory mediators like CRP, interleukine-6 (IL-6), and tumor necrosis factor alpha (TNF- $\alpha)^{46}$ leading to exacerbation of the inflammation.

Several observations sustain the hypothesis that visfatin may play a major role in the pathogenesis of RA. Some studies reported the upregulation of visfatin in activated RA in response to proinflammatory stimuli, such as IL- $6 .{ }^{47,48}$ Serum and synovial fluid Visfatin levels are correlated with the degree of inflammation, with the severity of the disease, and with joint damage. ${ }^{49}$ Circulating levels of visfatin correlate with obesity and are reduced 
after weight loss. ${ }^{50}$ Visfatin inhibition significantly reduced the severity of the disease in the experimental model of collagen-induced arthritis. ${ }^{51}$

In order to prevent the limitation in the effectiveness of the detection of the studied biomarkers to diagnoses the RA disease, Multivariate GLM analysis was used. This analysis depends on studying the detection limits of the studied biomarkers. The GLM analysis (Table 2) showed a high effect of being RA patients on the all measured biomarkers and excluding age and BMI as a covariate. The highest effect was on CRP followed by chemerin and UA respectively. While visfatin showed a small effect size on the diagnosis of RA disease. These results indicated clearly the inflammatory nature of RA disease. RA is characterized by inflammation and the formation of atherosclerosis can be exacerbated and accelerated by systemic inflammation. ${ }^{52}$ The inflammation occurred by the effect of certain cytokines that play a vital role in joint destruction and increases cellular infiltration in the synovium through enhancing chemokines expression. ${ }^{53}$

The correlation study showed many significant correlations among the measured parameters (Table 3). The most important correlations were between chemerin and many other parameters. These correlations may be due to many effects of chemerin on various biological processes. Chemerin regulates adipogenesis, angiogenesis and inflammation. ${ }^{54}$ Furthermore, serum levels of this adipokine are associated with components of metabolic syndrome, including increased body mass index (BMI), plasma triglyceride levels, and hypertension. ${ }^{55}$ Other study revealed that, in RA patients serum levels of chemerin are increased and they are more associated with systemic inflammation rather than obesity. ${ }^{56}$ These findings indicate chemerin as a useful biomarker of the disease activity. ${ }^{56}$ In the current study, there was also a significant positive correlation of chemerin with lipid profile (TC, LDL,VLDL,TG); while significant negative correlation with HDLc. High density lipoprotein function reversed cholesterol transport, and prevented LDL oxidation and thus reducing its atherogenic potential. ${ }^{57}$ Chemerin was suggested to play a role in the regulation of enzymes responsible for lipid metabolism by reducing the accumulation of adenosine cyclic monophosphate (cAMP) and stimulated calcium released in adipocytes. ${ }^{58}$ Moreover, other researchers have demonstrated a positive correlation between circulating visfatin levels and RA disease activity. ${ }^{59}$ There were a few studies about the effect of adipokine visfatin in RA which indicate a positive correlation between visfatin and inflammation markers, and elevated levels in RA patients when compared with control subjects. ${ }^{60,61} \mathrm{~A}$ 
significant increase of other parameters in the present study is in agreement with many studies deals with RA patients. ${ }^{62,63}$ The results also indicate a significant difference between RA patients and the control groups in Chemerin/Visfatin ratio. This gives another biomarker that can be used as a diagnostic factor since chemerin act as a pro-inflammatory adipokine. Visfatin level was found to be an inflammatory marker that associate with RA disease independently of CRP and uric acid. Rho et al (2009) demonstrated that serum visfatin level was positively correlated with age, BMI and inflammation markers (CRP, TNF- $\alpha$ and IL-6), with statistical significance in RA patients. ${ }^{64}$ Other studies have shown associations between visfatin and other inflammatory markers. ${ }^{58-65}$ In this study, another relationship was found between chemerin with CRP and UA, indicated a relationship with systemic inflammation. ${ }^{66}$ The inflammatory cytokines released by adipose tissue stimulate the synthesis of CRP in the liver, which was observed in inflamed tissues, in RA and in myocardium after infarction. ${ }^{67}$

In addition to the studied biomarkers for RA, Many other molecules were used as a diagnostic tool for diagnosis of RA with no absolute cut off values the can definitely refers to the disease. ${ }^{68}$ For studying the diagnostic accuracy of chemerin, visfatin and their ratio in the diagnosis of RA, ROC was constructed to obtain sensitivity, specificity, area under curve (AUC), of certain cut-off values of the biomarkers (Figure 1 and Table 4). ROC was chosen because it is superior to other commonly used methods and takes into account the correlation between sensitivity and 1-specificity at the cut-off values. The results showed relatively a good sensitivity and specificity of chemerin in diagnosis of RA (sensitivity=88.1 and specificity=75.9) in comparison with visfatin and chemerin/visfatin ratio. In comparison with other routine biomarkers used for diagnosis of RA, the sensitivity and specificity anti- cyclic citrullinated peptide antibody were $67 \%$ and $95 \%$, respectively and For IgM rheumatoid factor, the values were $69 \%$ and $85 \%$, respectively. ${ }^{69}$ This comparison indicated the ability of chemerin to diagnose RA efficiently but this diagnoses, due to relatively small specificity, may interfere with other inflammatory disorders. However, with adjuvant with other diagnostic parameters, chemerin may represent a useful addition in diagnosis of RA.

\section{Conclusions}

In conclusion, chemerin and visfatin levels seems to be linked to RA disease and can act as an inflammatory markers of RA patients. The present results suggest that serum 
chemerin and levels of chemerin and visfatin being symptomatic of RA and chemerin can be used for diagnosis of RA efficiently better than visfatin or chemerin to visfatin ratio. With adjuvant with other diagnostic parameters, chemerin may represent the future of diagnosis of RA efficiently and specifically.

Limitations of the study

The first limitation is the small samples size of the study. Therefore, research studies with much larger sample size would be required to ensure appropriate generalization of the findings of the study. The second limitation is the significance of the inter-assay $\mathrm{CV}$ of the ELISA kits that ranged between $3.97 \%-6.52 \%$ for chemerin and $10.83 \%-11.81 \%$ for visfatin kit.

\section{Acknowledgment}

We acknowledge the staff of Al-Sader Teaching Hospital in Al-Najaf province-Iraq for their assistance in the samples collection.

\section{Funding}

The manuscript was not funded officially.

\section{Conflict of interest}

The authors declared no conflict of interest.

Author's contributions

All authors have participated in the work and preparation of the manuscript. 


\section{References}

(1) Smolen JS, Aletaha D, McInnes IB. Rheumatoid arthritis. Lancet. 2016; 388(10055):2023-38.

(2) Bingham CO. III The pathogenesis of rheumatoid arthritis: pivotal cytokines involved in bone degradation and inflammation. J Rheumatol 2002; 29(65): 3-9.

(3) Gomez R, Conde J, Scotece M, Gomez-Reino JJ, Lago F, Gualillo O. What's new in our understanding of the role of adipokines in rheumatic disease? Nature Rev Rheumatol 2011; 7(9): 528-536.

(4) Kevin D. Deane1, M. Kristen Demoruelle1, Lindsay B. Kelmenson1, Kristine A. Kuhn1, Jill M. Norris2, and V. Michael Holers. Genetic and environmental risk factors for rheumatoid arthritis. Best Pract Res Clin Rheumatol. 2017; 31(1): 3-18.

(5) Gabriel SE, Crowson CS, OFFallon WM. The epidemiology of rheumatoid arthritis in Rochester, Minnesota, 1955-1985. Arthr Rhem. 1999; 42: 415-420.

(6) Alamonos Y, Drosos AA. Epidemiology of adult rheumatoid arthritis. Autoimmun Rev. 2005; 4: $130-136$.

(7) Lei Zhao, Yasuto Yamaguchi, Xiaomei Ge, William H. Robinson, John Morser and Lawrence L. K. Leung. Chemerin 156F, generated by chymase cleavage of prochemerin, is elevated in joint fluids of arthritis patients. Arthritis Res Ther 2018;20:132.

(8) Valcamonica E., C. Chighizola, D. Comi, O. De Lucia, L. Pisoni, A. Murgo, V. Salvi, S. Sozzani, P. Meroni. Levels of chemerin and interleukin 8 in the synovial fluid of patients with inflammatory arthritides and osteoarthritis. Clin Experl Rheumatol 2014; 32: 243-250.

(9) Al-Hakeim HK, Moustafa SR, Jasem KM. Serum Cesium, Rhenium, and Rubidium in Rheumatoid Arthritis Patients. Biol Trace Elem Res. 2019;189(2):379-386.

(10) Tolusso B, Alivernini S, Gigante MR, Ferraccioli G, Gremese E. Biomolecular features of inflammation in obese rheumatoid arthritis patients: management considerations. Exp Rev Clin Immunol. 2016; 22:1-12.

(11) Wittamer .V, Gregoire F, Robberecht P, Vassart G, Communi D, Parmentier M. The C-terminal nonapeptide of mature chemerin activates the chemerin receptor with low nanomolar potency. J Biol Chem 2004; 279(11): 9956-9962.

(12) Goralski K, McCarthy T, Hanniman E, Zabel B, Butcher E, Parlee S. Chemerin, a novel adipokine that regulates adipogenesis and adipocyte metabolism. J Biol Chem. 2007; 282: 28175-28188.

(13) Rourke JL, Dranse HJ, Sinal CJ. Towards an integrative approach to understanding the role of chemerin in human health and disease. Obesity Rev 2013; 14 245-262.

(14) Wittamer V, Franssen JD, Vulcano M, Mirjolet JF, Le Poul E, Migeotte I, Brezillon S, Tyldesley R, Blanpain C, Detheux M, et al. Specific recruitment of antigen-presenting cells by chemerin, a novel processed ligand from human inflammatory fluids. J Exp Med. 2003;198(7):977-85.

(15) Bozaoglu K, Bolton K, McMillan J, Zimmet P, Jowett J, Collier G, Walder K, Segal D. Chemerin is a novel adipokine associated with obesity and metabolic syndrome. Endocrinol 2007;148(10):4687-94.

(16) Takahashi M, Takahashi Y, Takahashi K, Zolotaryov FN, Hong KS, Kitazawa R, Iida K, Okimura Y, Kaji H, Kitazawa S, et al. Chemerin enhances insulin signaling and potentiates insulin-stimulated glucose uptake in 3T3-L1 adipocytes. FEBS Lett. 2008;582(5):573-8.

(17) Ouchi N, Parker JL, Lugus JJ, Walsh K. Adipokines in inflammation and metabolic disease. Nature Rev Immunol 2011; 11(2): 85-97.

(18) Hotamisligil GS. Inflammation and metabolic disorder. Nature. 2006; 444(7121): 860-867.

(19) Qin B, Yang M, Fu H, Ma N, Wei T, Tang Q, Hu Z, Liang Y, Yang Z, Zhong R. Body mass index and the risk of rheumatoid arthritis: a systematic review and dose-response meta-analysis. Arth Res Ther. 2015; 17:86-98.

(20) Moreno-Indias I, Oliva-Olivera W, Omiste A, Castellano-Castillo D, Lhamyani S, Camargo A, et al. Adipose tissue infiltration in normal-weight subjects and its impact on metabolic function. Transl Res. 2016;172:6-17.

(21) Klaasen R, Wijbrandts CA, Gerlag DM, Tak PP. Body mass index and clinical response to infliximab in rheumatoid arthritis. Arthritis Rheumatol. 2011; 63:359-64. 
(22) Sell H, Divoux A, Poitou C, Basdevant A, Bouillot JL, Bedossa P, Tordjman J, Eckel J, Clément K. Chemerin correlates with markers for fatty liver in morbidly obese patients and strongly decreases after weight loss induced by bariatric surgery. J Clin Endocrinol Metab. 2010;95:2892-6.

(23) Samal B, Sun Y, Stearns G, Xie C, Suggs S, McNiece I. Cloning and characterization of the cDNA encoding a novel human pre-b-cell colony-enhancing factor. Mol Cell Biol 1994; 14(2): 1431-1437.

(24) Lee YH, Bae SC. Circulating leptin level in rheumatoid arthritis and its correlation with disease activity: a meta-analysis. Z Rheumatol 2016;75(10):1021-1027

(25) Jacques C, Holzenberger M, Mladenovic Z. proinflammatory actions of visfatin/nicotinamide phosphoribosyltransferase (Nampt) involve regulation of insulin signaling pathway and Nampt enzymatic activity. J Biol Chem 2012; 287(18): 15100-15108.

(26) Rho YH, Solus J, Sokka T. Adipocytokines are associated with radiographic joint damage in rheumatoid arthritis. Arthritis Rheum 2009; 60:1906-1914.

(27) Giles JT, Allison M, Bingham CO 3rd, Scott WM Jr, Bathon JM. Adiponection is a mediator of the inverse association of adiposity with radiographic damage in rheumatoid arthritis. Arthritis Rheum 2009; 61:1248- 1256.

(28) Aletaha D, Neogi T, Silman A, Funovits J, Felson DT, Bingham CO 3rd, Birnbaum NS, Burmester GR, Bykerk VP, Cohen MD, et al. 2010 rheumatoid arthritis classification criteria: an American College of Rheumatology/European League Against Rheumatism collaborative initiative. Arthritis Rheum 2010;62(9):2569-2581.

(29) McClain DA, Abraham D, Rogers J, Brady R, Gault P, Ajioka R and Kushner J.P. Comparison of three methods for measuring high in rehabilitation in patients and the impact on body mass index classification: An open prospective study. Nutr Diet 2018; 75(1): 123-128.

(30) Giles JT, Ling SM, Ferrucci L, Bartlett SJ, Andersen RE, TownsM, et al. Abnormal body composition phenotypes in older rheumatoid arthritis patients: association with disease characteristics and pharmacotherapies. Arthritis Rheum. 2008;59:807-15.

(31) Skeoch S, Bruce IN. Atherosclerosis in rheumatoid arthritis: is it all about inflammation? Nat Rev Rheumatol. 2015;11(7):390-400.

(32) Yang X, Chang Y, Wei W. Endothelial dysfunction and inflammation: immunity in rheumatoid arthritis. Mediators Inflamm. 2016;2016:6813016.

(33) Brouwers H, von Hegedus J, Toes R, Kloppenburg M, Ioan-Facsinay A. Lipid mediators of inflammation in rheumatoid arthritis and osteoarthritis. Best Pract Res Clin Rheumatol. 2015;29(6):741-55.

(34) Neumann E, Junker S, Schett G, Frommer K, Müller-Ladner U: Adipokines in bone disease. Nat Rev Rheumatol 2016; 12: 296-302.

(35) Del Prete A, Salvi V, Sozzani S: Adipokines as potential biomarkers in rheumatoid arthritis. Mediators Inflamm 2014; 2014: 425068.

(36) Stejskal D, Karpisek M, Hanulova Z, Svestak M. Chemerin is an independent marker of the metabolic syndrome in a Caucasian population-a pilot study. Biomed Pap Med Fac Univ Palacky Olomouc Czech Repub. 2008; 152: 217-21.

(37) Lchrke M, Becker A, Greif M, Stark R, Laubender RP, Von Ziegler F. Chemerin is associated with markers of inflammation and components of the metabolic syndrome but does not predict coronary atherosclerosis. Eur J Endocrinol 2009; 161: 339-44.

(38) El-Mesallamy Ho, El-Derany Mo, Hamdy NM. Serum omentin-1 and chemerin levels are interrelated in patients with Type 2 diabetes mellitus with or without ischaemic heart disease. Diabet Med 2011; 28: 1194-200.

(39) Fatima SS, Rehman R, Baig M, Khan TA. New Roles of the multidimensional adipokine: chemerin. Peptides. 2014; 62: 15-20.

(40) Ernst MC, Sinal CJ. Chemerin: at the crossroads of inflammation and obesity. Trends Endocrinol Metab. 2012; 21: 660-667.

(41) Duraiswamy A, Shanmugasundaram D, Sasikumar CS, Cherian K M. Chemerin: a potential target in coronary artery disease-a review. IJBAR. 2012; 3: 537-540.

(42) Bozaoglu K, Curran JE, Stocker CJ, Zaibi MS, Segal D, Konstantopoulos N. Chemerin, a novel adipokine in the regulation of angiogenesis. J Clin Endocrinol Metab. 2010; 95: 2476-2485. 
(43) Zabel BA, Allen SJ, Kulig P, Allen JA, Cichy J, Handel TM. Chemerin activation by serine proteases of the coagulation, fibrinolytic, and inflammatory cascades. J Biol Chem 2005; 280 : 34661-34666.

(44) Fontesa VS, Nevesa FS, Cândido APC. Chemerin and factors related to cardiovascular risk in children and adolescents: a systematic review. Rev Paul Pediatr 2018;36(2):221-229.

(45) Rourke JL, Dranse HJ, Sinal CJ. Towards an integrative approach to understanding the role of chemerin in human health and disease. Obes Rev 2013; 14: 245-62.

(46) Roman AA, Parlee SD, Sinal CJ. Chemerin: a potential endocrine link between obesity and type 2 diabetes. Endocrine 2012; 42: 243-51.

(47) Brentano F, Schorr O, Ospelt C, Stanczyk J, Gay RE, Gay S, Kyburz D. Pre-B cell colonyenhancing factor/visfatin, a new marker of inflammation in rheumatoid arthritis with proinflammatory and matrix-degrading activities. Arthritis Rheum 2007;56(9):2829-2839.

(48) Sglunda O, Mann H, Hulejová H, Kuklová M, Pecha O, Pleštilová L, Filková M, Pavelka K, Vencovský J, Senolt L. Decreased circulating visfatin is associated with improved disease activity in early rheumatoid arthritis: data from the PERAC cohort. PLoS One. 2014;9(7):e103495.

(49) Klein-Wieringa IR, van der Linden MPM, Knevel R, et al. Baseline serum adipokine levels predict radiographic progression in early rheumatoid arthritis. Arthritis Rheum 2011;63(9):2567-2574.

(50) Haider DG, Schindler K, Schaller G, Prager G, Wolzt M, Ludvik B. Increased plasma visfatin concentrations in morbidly obese subjects are reduced after gastric banding. J Clin Endocrinol Met 2006;91(4):1578-1581.

(51) Evans L, Williams AS, Hayes AJ, Jones SA, Nowell M. Suppression of leukocyte infiltration and cartilage degradation by selective inhibition of pre-B cell colony-enhancing factor/visfatin/nicotinamide phosphoribosyltransferase: apo866-mediated therapy in human fibroblasts and murine collagen-induced arthritis. Arthritis Rheum 2011;63(7):1866-1877.

(52) Ku I. A., Imboden J. B., Hsue P. Y., Ganz P. Rheumatoid arthritis: a model of systemic inflammation driving atherosclerosis. Circulation Journal. 2009;73(6):977-985.

(53) Yap HY, Tee SZ, Wong MM, Chow SK, Peh SC, Teow SY. Pathogenic Role of Immune Cells in Rheumatoid Arthritis: Implications in Clinical Treatment and Biomarker Development. Cells. 2018;7(10). pii: E161.

(54) Lu B, Zhao M, Jiang W, Ma J, Yang C, Shao J, Gu P. Independent association of circulating level of chemerin with functional and early morphological vascular changes in newly diagnosed type 2 diabetic patients. Medicine (Baltimore) 2015; 94: e1990

(55) Lehrke M, Bscker A, Greif M Stark R, Laubender RP, von Ziegler F, Lebherz C, Tittus J, Reiser M, Becker C, Göke B, Leber AW, Parhofer KG, Broedl UC. Chemerin is associated with markers of inflammation and components of the metabolic syndrome but does not predict coronary atherosclerosis. Eur J Endocrinol 2009; 161: 339-44.

(56) Tolusso B, Alivernini S, Gigante MR, Ferraccioli G, Gremese E: Biomolecular features of inflammation in obese rheumatoid arthritis patients: management considerations. Expert Rev Clin Immunol 2016; 12: 751-62

(57) Ernst MC, Issa M, Goralski KB, Sinal CJ. Chemerin exacerbates glucose intolerance in mouse models of obesity and diabetes. Endocrinol 2010; 152: 1998-2007.

(58) Maghsoudi Z, Kelishadi R, Hosseinzadeh-Attar MJ. Association of chemerin levels with anthropometric indexes and C-reactive protein in obese and non-obese adolescents. Diabetes Metab Syndr. 2016; 10(Suppl.1): S43-6.

(59) Lee YH, Bae SC. Circulating adiponectin and visfatin levels in rheumatoid arthritis and their correlation with disease activity: A meta-analysis. Int. J Rheum Dis 2018;21: 664-672.

(60) Rho YH, Solus J, Sokka J. Adipocytokines are associated with radiographic joint damage in rheumatoid arthritis. Arthritis Rheum 2009; 60: 1906-1914.

(61) Robinson C, Tsang L, Solomon A, Woodiwiss AJ, Gunter S, Mer M, Hsu HC, Gomes M, Norton GR, Millen AME, Dessein PH. Nesfatin-1 and visfatin expression is associated with reduced atherosclerotic disease risk in patients with rheumatoid arthritis. Peptides 2018;102:3137. 
(62) Chavan VU, Ramavataram DVSS, Patel PA, Rupani MP. Evaluation of Serum Magnesium, Lipid Profile and Various Biochemical Parameters as Risk Factors of Cardiovascular Diseases in Patients with Rheumatoid Arthritis. J Clin Diagnos Res 2015;9(4): BC01-BC05.

(63) George Steiner, Murray B.Urowitz. Lipid profiles in patients with rheumatoid arthritis: Mechanisms and the Impact of Treatment. Semin Arthritis Rheum 2009; 38(5) 372-381

(64) Tilg H, Moschen Ar. Adipocytokines: Mediators linking adipose tissue inflammation and immunity. Nat Rev Immunol 2006; 6:772-783.

(65) Luk T, Malam Z, Marshall JC. Pre-B cell colony-enhancing factor (PBEF)/ Visfatin: a novel mediator of innate immunity. J Leukoc Biol. 2008; 83: 804-16.

(66) Landgraf K, Friebr D, Ullrich T, Kratzsch J, Dittrich K, Herberth G. Chemerin as a mediator between obesity and vascular inflammation in children. J Clin Endocrinol Metab 2012; 97 : E556-64.

(67) Martins LM, Oliveira AR, Cruz KJ, Torres-Leal FL, Marreiro DN. Obesity, inflammation, and insulin resistance. Braz J Pharm Sci 2014; 50: 677-92.

(68) Atzeni F, Talotta R, Masala IF, Bongiovanni S, Boccassini L, Sarzi-Puttini P. Biomarkers in Rheumatoid Arthritis. Isr Med Assoc J. 2017 Aug;19(8):512-516.

(69) Nishimura K, Sugiyama D, Kogata Y, Tsuji G, Nakazawa T, Kawano S, Saigo K, Morinobu A, Koshiba M, Kuntz KM, Kamae I, Kumagai S. Meta-analysis: diagnostic accuracy of anticyclic citrullinated peptide antibody and rheumatoid factor for rheumatoid arthritis. Ann Intern Med. 2007;146(11):797-808. 


\section{LEGENDS}

Table 1: Socio-demographic, clinical and biomarker data in RA patients as compared with healthy controls.

Table 2. Results of multivariate GLM analysis with the biomarkers as dependent variables and diagnosis as explanatory variable while adjusting for extraneous variables.

Table 3. Inter-correlation matrix of the measured parameters.

Table 4. Data of ROC curve, AUC, sensitivity, and specificity of Chemerin, visfatin, and their ration in the diagnosis of $\mathrm{RA}$.

Figure 1: ROC of the A: Chemerin, B: Chemerin/Visfatin, \& C: Visfatin for the diagnosis of RA. 
Table 1: Socio-demographic, clinical and biomarker data in RA patients as compared with healthy controls.

\begin{tabular}{|c|c|c|c|}
\hline Parameter & Patients & Control & p-value \\
\hline$\left(\mathrm{Kg} / \mathrm{m}^{2}\right)$ & $28.11 \pm 2.27$ & $27.45 \pm 1.24$ & 0.081 \\
\hline (years) & $40.05 \pm 7.99$ & $40.83 \pm 8.47$ & 0.675 \\
\hline$(\mathrm{mg} / \mathrm{L})$ & $8.23 \pm 1.62$ & $5.26 \pm 0.99$ & $<0.001$ \\
\hline$(\mathrm{mg} / \mathrm{dl})$ & $6.45 \pm 1.47$ & $4.88 \pm 0.79$ & $<0.001$ \\
\hline Visfatin $\quad(\mathrm{ng} / \mathrm{ml})$ & $43.02 \pm 11.72$ & $36.67 \pm 9.58$ & 0.013 \\
\hline Chemerin (ng/ml) & $239.34 \pm 49.86$ & $174.32 \pm 34.82$ & $<0.001$ \\
\hline Chemerin/Visfatin & $5.88 \pm 1.69$ & $5.09 \pm 0.1 .67$ & 0.041 \\
\hline$(\mathrm{mmol} / \mathrm{L})$ & $4.65 \pm 0.82$ & $4.19 \pm 0.89$ & 0.019 \\
\hline$(\mathrm{mmol} / \mathrm{L})$ & $1.36 \pm 0.15$ & $1.27 \pm 0.13$ & 0.005 \\
\hline VLDLc $(\mathrm{mmol} / \mathrm{L})$ & $0.62 \pm 0.07$ & $0.58 \pm 0.06$ & 0.005 \\
\hline HDLc $\quad(\mathrm{mmol} / \mathrm{L})$ & $0.95 \pm 0.16$ & $1.15 \pm 0.12$ & $<0.001$ \\
\hline$(\mathrm{mmol} / \mathrm{L})$ & $3.08 \pm 0.85$ & $2.27 \pm 0.96$ & 0.003 \\
\hline TC/HDLc & $5.08 \pm 1.41$ & $3.75 \pm 1.10$ & $<0.001$ \\
\hline TG/HDLc & $1.48 \pm 0.33$ & $1.12 \pm 0.19$ & $<0.001$ \\
\hline LDL/HDLc & $3.40 \pm 1.32$ & $2.24 \pm 1.05$ & $<0.001$ \\
\hline
\end{tabular}

BMI: Body mass index; CRP:C-reactive protein; UA: Uric Acid; TC: Total cholesterol; TG: Triglyceride; VLDLc: Very low density lipoprotein-cholesterol; HDLc: High density lipoproteincholesterol; LDLc: Low density lipoprotein-cholesterol. 
Table 2. Results of multivariate GLM analysis with the biomarkers as dependent variables and diagnosis as explanatory variable while adjusting for extraneous variables.

\begin{tabular}{|c|c|c|c|c|c|c|}
\hline Tests & $\begin{array}{l}\text { Dependent } \\
\text { Parameters }\end{array}$ & $\begin{array}{c}\text { Explanatory } \\
\text { variables }\end{array}$ & $\mathrm{F}$ & $\mathrm{df}$ & $\mathrm{p}$-value & $\begin{array}{c}\text { Partia } \\
\eta^{2}\end{array}$ \\
\hline \multirow[t]{3}{*}{ Multivariate } & \multirow{3}{*}{$\begin{array}{l}\text { All } \\
\text { biomarkers }\end{array}$} & Diagnosis & $18.117^{b}$ & $10 / 79$ & $<0.001$ & 0.707 \\
\hline & & BMI & $0.900^{\mathrm{b}}$ & $10 / 79$ & 0.054 & 0.107 \\
\hline & & Age & $1.140^{\mathrm{b}}$ & $10 / 79$ & 0.071 & 0.075 \\
\hline \multirow{13}{*}{$\begin{array}{c}\text { Tests of } \\
\text { Between- }\end{array}$} & \multirow{13}{*}{ Diagnosis } & CRP & 77.644 & 1 & $<0.001$ & 0.48 \\
\hline & & UA & 28.317 & 1 & $<0.001$ & 0.252 \\
\hline & & Visfatin & 7.234 & 1 & 0.009 & 0.079 \\
\hline & & Chemerin & 35.113 & 1 & $<0.001$ & 0.295 \\
\hline & & Chemrin/Visfatin & 3.2 & 1 & 0.077 & 0.037 \\
\hline & & $\mathrm{TC}$ & 5.757 & 1 & 0.019 & 0.064 \\
\hline & & TG & 7.261 & 1 & 0.009 & 0.08 \\
\hline & & VLDLc & 7.261 & 1 & 0.009 & 0.08 \\
\hline & & HDLc & 9.882 & 1 & $<0.001$ & 0.081 \\
\hline & & LDLc & 9.151 & 1 & 0.003 & 0.098 \\
\hline & & TC/HDLc & 18.677 & 1 & $<0.001$ & 0.182 \\
\hline & & TG/HDLc & 17.818 & 1 & $<0.001$ & 0.149 \\
\hline & & LDL/HDLc & 16.229 & 1 & $<0.001$ & 0.162 \\
\hline
\end{tabular}

BMI: Body mass index; CRP:C-reactive protein; UA: Uric Acid; TC: Total cholesterol; TG: Triglyceride; VLDLc: Very low density lipoprotein-cholesterol; HDLc: High density lipoproteincholesterol; LDLc: Low density lipoprotein-cholesterol. 
Table 3. Inter-correlation matrix of the measured parameters.

\begin{tabular}{|l|c|c|c|c|c|}
\hline Parameter & CRP & UA & Visfatin & Chemerin & Chemerin/Visfatin \\
\hline UA & $0.340^{* *}$ & & & & \\
\hline Visfatin & 0.113 & 0.042 & & & \\
\hline Chemerin & $0.433^{* *}$ & $0.291 * *$ & $0.328 * *$ & & \\
\hline Chemerin/Visfatin & $0.270^{*}$ & 0.195 & $-0.599 * *$ & $0.513 * *$ & \\
\hline BMI & $0.241^{*}$ & 0.162 & -0.021 & 0.173 & 0.171 \\
\hline Age & -0.155 & 0.136 & 0.166 & -0.127 & $-0.260 *$ \\
\hline TC & 0.134 & 0.067 & 0.152 & $0.233^{*}$ & 0.067 \\
\hline TG & $0.241^{*}$ & 0.153 & 0.176 & 0.106 & -0.061 \\
\hline VLDLc & $0.241^{*}$ & 0.153 & 0.176 & 0.106 & -0.061 \\
\hline HDLc & $-0.342^{* *}$ & $-0.236 *$ & -0.162 & $-0.243^{*}$ & -0.044 \\
\hline LDLc & 0.172 & 0.096 & 0.159 & $0.256^{*}$ & 0.076 \\
\hline TC/HDLc & $0.257 *$ & 0.145 & 0.151 & $0.243 *$ & 0.058 \\
\hline TG/HDLc & $0.325^{* *}$ & $0.217 *$ & 0.166 & 0.172 & -0.019 \\
\hline LDL/HDLc & $0.240^{*}$ & 0.132 & 0.144 & $0.242^{*}$ & 0.065 \\
\hline
\end{tabular}

* Correlation is significant at the 0.05 level. $* *$ Correlation is significant at the 0.01 level. 
Table 4. Data of ROC curve, AUC, sensitivity, and specificity of Chemerin, visfatin, and their ration in the diagnosis of RA.

\begin{tabular}{|l|c|c|c|c|c|c|}
\hline Variables & $\begin{array}{c}\text { Youdin J } \\
\text { statistic }\end{array}$ & AUC & Cut-off value & $\mathrm{p}$ & Sensitivity & Specificity \\
\hline Visfatin & 0.32 & 0.663 & $39.02 \mathrm{ng} / \mathrm{ml}$ & 0.013 & 64.4 & 58.6 \\
\hline Chemerin & 0.72 & 0.885 & $187.88 \mathrm{ng} / \mathrm{ml}$ & $<0.001$ & 88.1 & 75.9 \\
\hline Chemerin/Visfatin & 0.37 & 0.649 & 5.03 & 0.023 & 66.1 & 65.5 \\
\hline
\end{tabular}




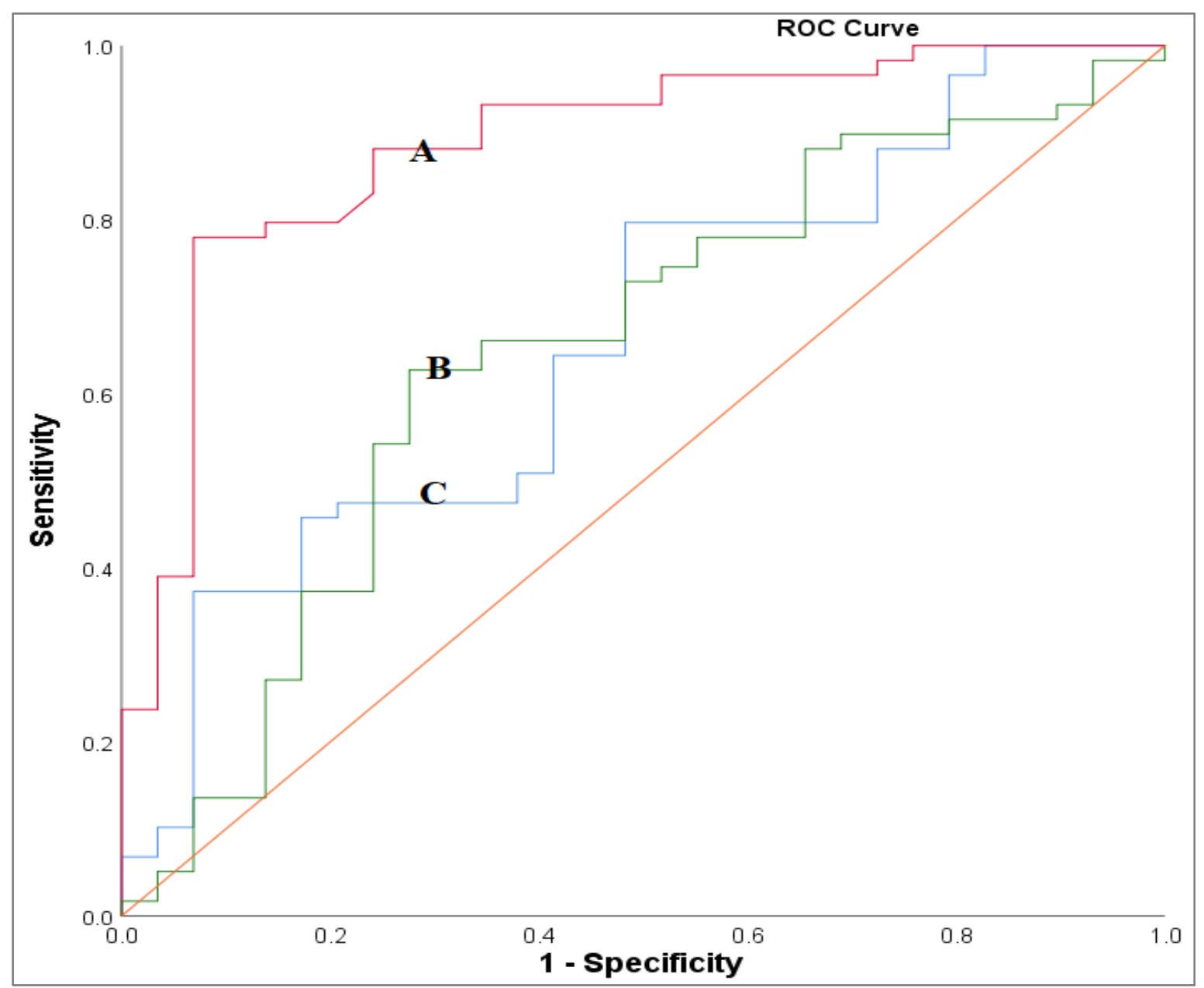

Figure 1: ROC of the A: Chemerin, B: Chemerin/Visfatin, \& C: Visfatin for the diagnosis of RA. 